\title{
Association of insulin resistance indexes QUICKI and HOMA-IR with the parameters of metabolic syndrome in non-diabetes Japanese male population
}

\author{
Ayako Kakita ${ }^{1,2)}$, Atsushi Suzuki ${ }^{2)}$, Mutsuko Nagata ${ }^{2)}$, Mitsuyasu Itoh ${ }^{2)}$ \\ 1) The Kenwakai Medical Foundation Marine Clinic \\ 2) Division of Endocrinology and Metabolism, Department of Internal Medicine, Fujita Health University
}

\begin{abstract}
The metabolic syndrome is a common and complex disorder combining obesity, dyslipidemia, hypertension, and insulin resistance (IR). In the present study, we conducted the observational study in 126 non-diabetes Japanese male population, who visited our clinic for their routine medical check-up, about the relationship between IR indexes and parameters of metabolic syndrome. IR was assessed by both quantitative insulin check index (QUICKI) and homeostasis model assessment for IR (HOMA-IR). Body mass index, body fat content and blood pressure were related with QUICKI and HOMA-IR, but better association was observed with QUICKI. There was no significant association between age and IR indexes. In addition to the fasting plasma glucose (PG) and immunoreactive insulin (IRI) levels, the concentrations of PG and IRI at 30, 60 and 120 min during $75 \mathrm{~g}$ oral glucose tolerance test were associated with HOMA-IR and QUICKI. Serum triglyceride concentration was associated with QUICKI and HOMA-IR, and serum HDL-cholesterol was related to QUICKI. On the contrary, none of serum total cholesterol, LDL-cholesterol or non-HDL cholesterol concentration was associated with IR indexes. These results suggest that IR was significantly related with the progress of metabolic syndrome even in non-diabetes Japanese male population. Early evaluation of IR by QUICKI would be expected to contribute to the prevention of cardiovascular event due to metabolic syndrome.
\end{abstract}

KEY WORDS: insulin resistance, metabolic syndrome, obesity, hypertension

\section{Introduction}

Insulin resistance, decreased sensitivity or responsiveness to the metabolic actions of insulin, is associated with not only type 2 diabetes mellitus but also a number of other diseases, including hypertension, dyslipidemia, visceral fat obesity, and coronary artery disease, or metabolic syndrome ${ }^{1-3}$. The significant decline of insulin action has been reported with aging, suggesting the possible association between aging and insulin resistance ${ }^{4)}$. Several methods are available to assess insulin sensitivity in humans. Among them, the golden standard method is so far the euglycemic hyperinsulinemic clamp, because it directly measures the insulin action on glucose utilization under steady-state condition ${ }^{5)}$. However, this technique is laboratorious and only applicable to a small number of subjects at specialized institution for diabetes. Therefore, the ability to easily quantify insulin resistance in large number of non-diabetes people may be useful for the early diagnosis of these diseases ${ }^{6-8)}$. Various methods including homeostasis model assessment of insulin resistance $(\text { HOMA-IR })^{9)}$ and the quantitative insulin sensitivity check index (QUICKI) ${ }^{10)}$ are proposed to evaluate insulin resistance. HOMAIR and QUICKI, which are derived from fasting plasma glucose (PG) and immunoreactive insulin (IRI) levels, are considered to reflect insulin resistance, although the more precise validation of these indexes to evaluate insulin resistance is still needed Metabolic syndrome is now well accepted to be a clinical tool for identifying individuals predisposed to diabetes and adverse cardiovascular outcomes ${ }^{11-13)}$. The pathogenetic mechanism underlying the metabolic syndrome remains uncertain, although central obesity and insulin resistance have been proposed to play causative roles. Asians generally have less body mass index (BMI) than Caucasians in developed countries, but are thought to have a higher body fat percentage and cardiovascular risks than Caucasians at a given BMI ${ }^{14)}$. So, it seems necessary to evaluate their insulin resistance and its association with metabolic factors.

The aim of this study is to analyze the relationship between insulin resistance indexes and parameters of metabolic syndrome in non-diabetes Japanese male population. 


\section{Materials and Methods}

\section{Subjects}

Subjects were non-diabetes population who visited Marine Clinic for their routine medical check-up, and agreed to participate in this study. In Marine Clinic, about 70 subjects visit for medical check-up and health evaluation everyday. The participants were the office workers and their housewives, and their employer generally covers the cost of medical check-up. Subject who has present or past history of diabetes mellitus, any medication for hypertension, dyslipidemia or diabetes was excluded from this study. The subjects, whose blood HbAlc level was equal to or more than $6.5 \%$, were also excluded from this study. After that, we conducted $75 \mathrm{~g}$ oral glucose tolerance test (OGTT) on 140 Japanese male subjects. As for female population, the number of the subjects $(\mathrm{n}=12)$ who were conducted $75 \mathrm{gOGTT}$ was too small to be statistically analyzed. As the criteria of metabolic syndrome in male is different from that in female, we showed the data only in male population in this study. Then the subjects whose fasting PG was equal to or more than $126 \mathrm{mg} / \mathrm{dl}$ were excluded. In addition, the subjects whose PG at 120 min during $75 \mathrm{~g}$ OGTT was equal to or more than $200 \mathrm{mg} / \mathrm{dl}$ were also excluded from this study. Finally, 126 non-diabetes Japanese male subjects were enrolled to this study.

\section{Methods}

Height, weight and body fat content were measured by the bioelectrical impedance analysis method using Bodyfat Analyzer TBF-202 (Tanita Inc., Tokyo, Japan). Systolic and diastolic blood pressure was examined by automatic sphygmomanometer three times for each patient, and was calculated their average. After a 12-h overnight fast, a fasting blood sample was taken for the determination of fasting PG and fasting IRI. PG levels were measured by the glucose oxidase method, blood HbAlc by stepwise high-pressure liquid chromatography, and plasma IRI levels by chemiluminescent enzyme immunoassay. Routine chemistries were measured by enzymatic methods adapted to an Olympus AU5232 automatic analyzer (Olympus Co., Tokyo, Japan). The hospital's ethics review committee approved this descriptive study. Informed consent was obtained in compliance with Declaration of Helsinki.

\section{$75 \mathrm{~g}$ OGTT and blood samples}

A standard $75 \mathrm{~g}$ OGTT was performed after a 12 -h overnight fast. Plasma samples were obtained at $0,30,60$, and 120 min after glucose loading. The concentrations of PG and IRI levels were measured at each time point. At the same time, blood $\mathrm{HbAlc}$ level, serum total cholesterol (TC), serum triglyceride (TG), low density lipoprotein-cholesterol (LDL-C) and high density lipoprotein -cholesterol (HDL-C) concentrations were measured at $0 \mathrm{~min}$.

\section{HOMA-IR and QUICKI}

HOMA-IR was used to calculate an index from the product of the fasting concentrations of IRI $(\mu \mathrm{U} / \mathrm{ml})$ and PG $(\mathrm{mg} / \mathrm{dl})$ divided by $405^{9)}$. The QUICKI was calculated as follows: $1 /[\log$ (fasting IRI) $+\log (\text { fasting PG) }]^{10)}$.

\section{Statistical analysis}

The relationship between HOMA-IR and QUICKI as dependent variables and blood HbA1c, PG, IRI, serum TC, LDL-C, HDL-C, non-HDL-C, TG concentrations, body weight, BMI, body fat content, blood pressure as independent variables were examined using single regression analysis (Stat View version 5.0, SAS Inc, Cary, NC, USA). The data was considered to be significant when the $\mathrm{P}$ value was $<0.05$.

\section{Results}

The clinical characteristics of the subjects are shown in Table 1. Total number of the study population was 126 , aged $30-66$ years (Mean $\pm \mathrm{SD}, 47.5 \pm 8.2$ ). Mean BMI was $23.9 \pm 3.2 \mathrm{~kg} / \mathrm{m}^{2}$ (range $15.0-36.3$ ), and mean body fat content was $21.4 \pm 5.4 \%$ (range 10.4-44.1). Mean HbA1c was $5.16 \pm 0.41 \%$ (range 4.3-6.4). The average HOMA-IR and QUICKI were $1.62 \pm 1.08$ and $0.36 \pm 0.03$, respectively. The result of $75 \mathrm{~g}$ OGTT showed that mean fasting PG level was $96.7 \pm 11.2 \mathrm{mg} / \mathrm{dl}$, and mean PG at $120 \mathrm{~min}$ was $113.6 \pm 36.1 \mathrm{mg} / \mathrm{dl}$, while fasting IRI and IRI at $120 \mathrm{~min}$ were 6.7 \pm 4.4 and $32.7 \pm 23.7 \mu \mathrm{U} / \mathrm{ml}$, respectively. QUICKI and HOMAIR were related with BMI, body fat content and systolic blood pressure, but not with age (Table 2). Better association was observed with QUICKI. In addition, diastolic pressure was related to not HOMA-IR but QUICKI. The concentrations of PG and IRI at $0,30,60$ and $120 \mathrm{~min}$ during $75 \mathrm{~g}$ OGTT were also associated with HOMA-IR and QUICKI (Table 3). TG was related with both QUICKI and HOMA-IR, and HDL-C was associated with QUICKI, even though their serum TG and HDL-C concentrations were within normal range in the majority of this population ( $\mathrm{TG}<150,91 / 126$; HDL-C $\geq 40,104 / 126)$. However, these insulin resistance indexes were not related with TC or LDL-C (Figure 1 and 2). Furthermore, non-HDL-C was not associated with either HOMA-IR or QUICKI ( $\mathrm{R}=0.047, \mathrm{p}=0.603$ for HOMA-IR; $\mathrm{R}=0.091, \mathrm{p}=0.311$ for QUICKI). In this study, HbA1c had significant correlation with QUICKI but not with HOMA-IR (QUICKI: $\mathrm{R}=0.215, \mathrm{p}=0.016$; HOMA-IR: $\mathrm{R}=0.125, \mathrm{P}=0.162$ ).

\section{Table 1 Details of study subjects}

\begin{tabular}{lc}
\hline Variable & \\
\hline Total number of the subjects & 126 \\
Age(year) & $47.5 \pm 8.2$ \\
Body Mass Index $\left(\mathrm{kg} / \mathrm{m}^{2}\right)$ & $23.9 \pm 3.2$ \\
Body fat content $(\%)$ & $21.4 \pm 5.4$ \\
HbAlc $(\%)$ & $5.16 \pm 0.41$ \\
SBP $(\mathrm{mmHg})$ & $122.0 \pm 15.9$ \\
DBP(mmHg) & $72.9 \pm 12.7$ \\
TC $(\mathrm{mg} / \mathrm{dl})$ & $201.9 \pm 29.9$ \\
TG(mg/dl) & $130.5 \pm 92.9$ \\
HDL-C(mg/dl) & $52.5 \pm 14.4$ \\
LDL-C(mg/dl) & $120.0 \pm 30.9$ \\
Non HDL-C(mg/dl) & $149.4 \pm 32.9$ \\
HOMA-IR & $1.62 \pm 1.08$ \\
QUICKI & $0.36 \pm 0.03$ \\
\hline
\end{tabular}

SBP, systolic blood pressure; DBP, diastolic blood pressure; TC, total cholesterol; TG, triglyceride; HDL-C, high-density lipoprotein-cholesterol; LDL-C, low-density lipoprotein cholesterol; HOMA-IR, homeostasis model assessment for insulin resistance; QUICKI, quantitative insulin check index. 
Table 2

\begin{tabular}{l|c|c|c|r}
\hline & \multicolumn{2}{|c|}{ QUICKI } & \multicolumn{2}{c}{ HOMA-IR } \\
\hline & $\mathrm{R}$ & \multicolumn{1}{c|}{$P$} & $\mathrm{R}$ & \multicolumn{1}{c}{$P$} \\
\hline Age (years) & 0.050 & 0.573 & 0.041 & 0.648 \\
Body Mass Index (kg/m²) & 0.434 & $<0.001$ & 0.365 & $<0.001$ \\
Body fat content (\%) & 0.440 & $<0.001$ & 0.320 & $<0.001$ \\
Systolic BP (mmHg) & 0.329 & $<0.001$ & 0.213 & 0.016 \\
Diastolic BP (mmHg) & 0.250 & 0.005 & 0.125 & 0.163 \\
\hline
\end{tabular}

BP, blood pressure
Table 3

\begin{tabular}{l|c|c|c|r}
\hline & \multicolumn{2}{|c|}{ QUICKI } & \multicolumn{2}{c}{ HOMA-IR } \\
\hline & $\mathrm{R}$ & $P$ & $\mathrm{R}$ & \multicolumn{1}{c}{$P$} \\
\hline PG 0 min & 0.482 & $<0.001$ & 0.320 & $<0.001$ \\
PG 30 min & 0.380 & $<0.001$ & 0.363 & $<0.001$ \\
PG 60 min & 0.293 & $<0.001$ & 0.197 & 0.027 \\
PG 120 min & 0.293 & $<0.001$ & 0.221 & 0.013 \\
IRI 0 min & 0.798 & $<0.001$ & 0.983 & $<0.001$ \\
IRI 30 min & 0.386 & $<0.001$ & 0.682 & $<0.001$ \\
IRI 60 min & 0.443 & $<0.001$ & 0.575 & $<0.001$ \\
IRI 120 min & 0.605 & $<0.001$ & 0.626 & $<0.001$ \\
\hline
\end{tabular}

PG, plasma glucose; IRI, immunoreactive insulin
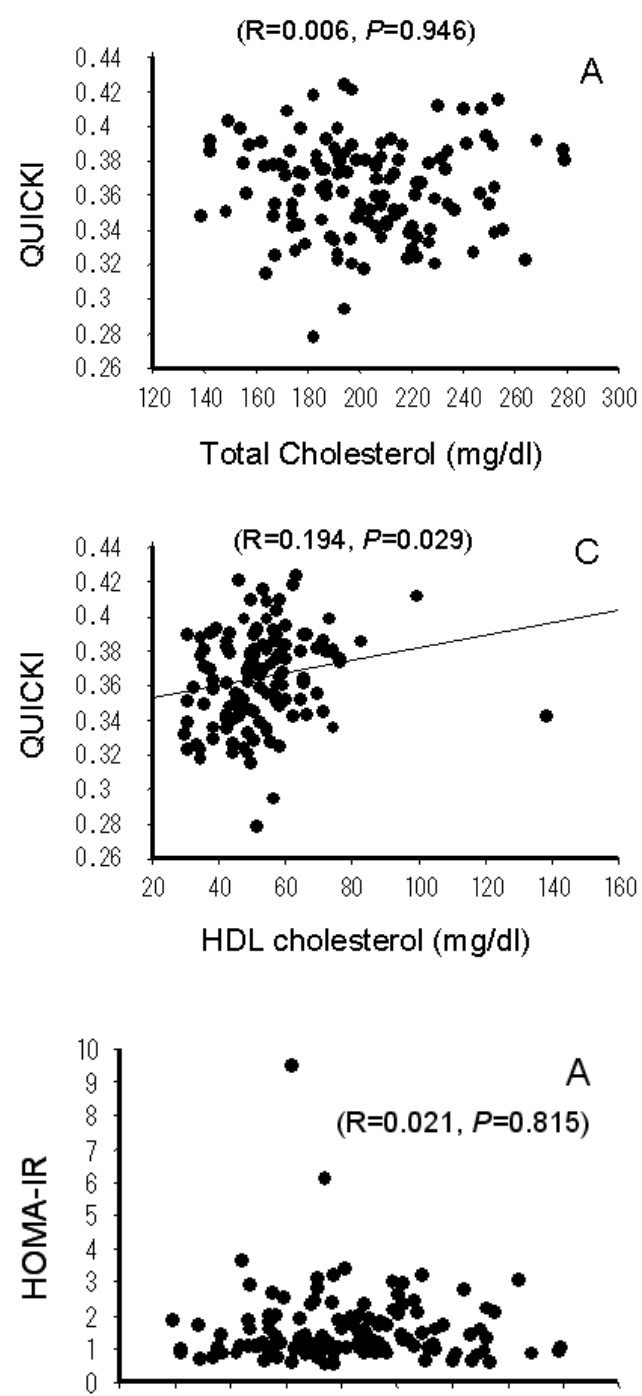

$\begin{array}{llllllllll}120 & 140 & 160 & 180 & 200 & 220 & 240 & 260 & 280 & 300\end{array}$

Total Cholesterol (mg/dl)

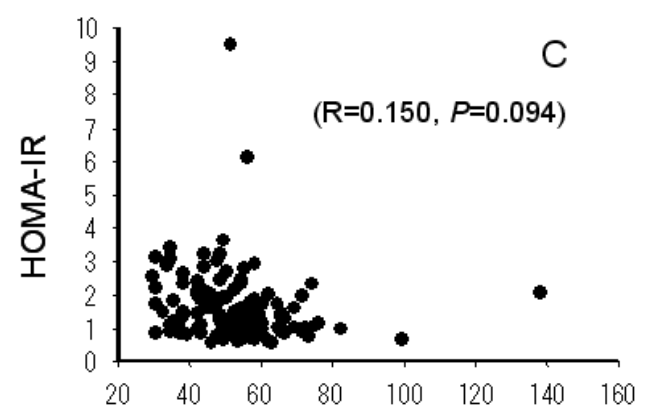

HDL cholesterol (mg/dl)
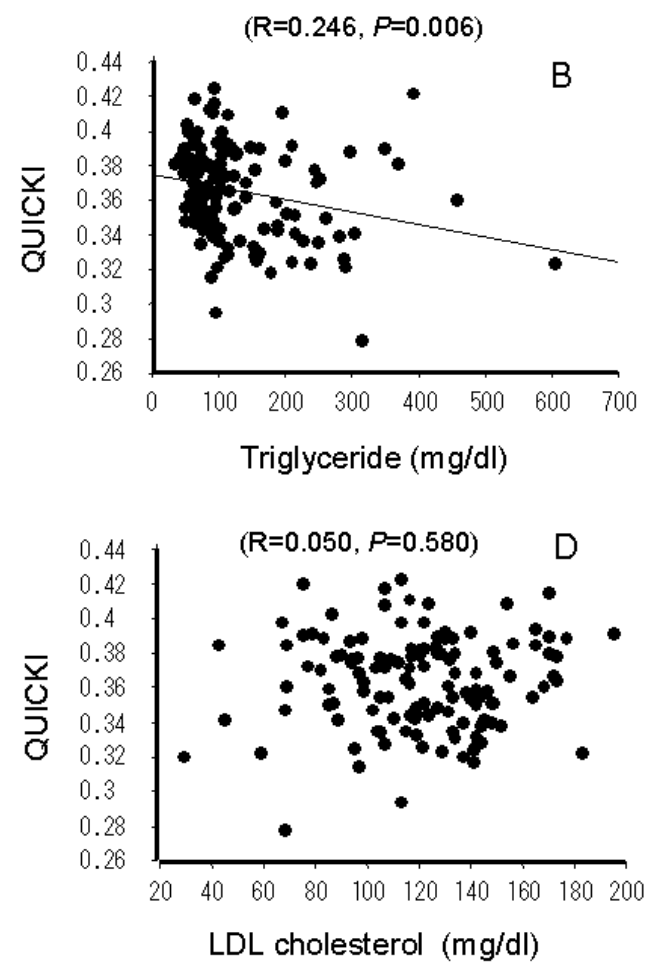

Fig. 1

Relationship between quantitative insulin check index (QUICKI) and serum total cholesterol (A), triglyceride (B), highdensity lipoprotein (HDL)-cholesterol(C) and low-density lipoprotein (LDL)cholesterol (D) concentrations.

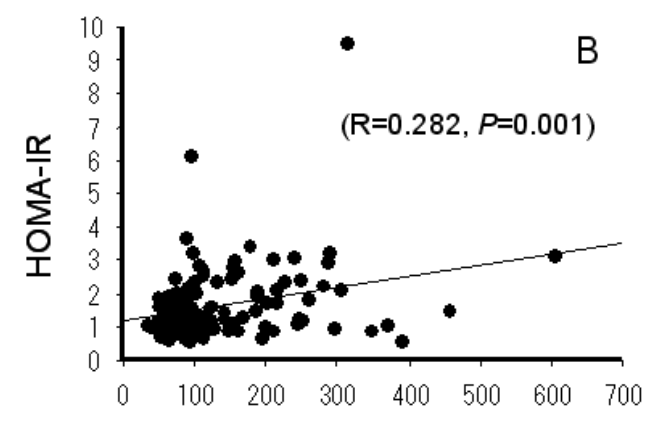

Triglyceride $(\mathrm{mg} / \mathrm{dl})$

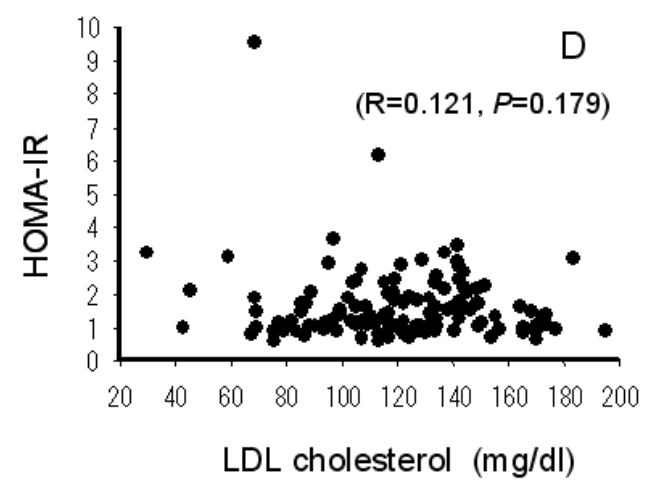

Fig. 2

Relationship between homeostasis model assessment for IR (HOMA-IR) and serum total cholesterol (A), triglyceride (B), highdensity lipoprotein (HDL)-cholesterol(C) and low-density lipoprotein (LDL)cholesterol (D) concentrations. 


\section{Discussion}

Metabolic syndrome is now well known as a causative condition for the development of chronic diseases such as diabetes, hypertension and dyslipidemia, all of which result in the lifethreatening cardiovascular diseases ${ }^{2,15}$. The combination of insulin resistance and compensatory hyperinsulinemia increases the likelihood that an individual will be hypertensive, and has a dyslipidemia characterized by high serum TG and low HDL-C concentrations ${ }^{16)}$. Some therapies including weight reduction, exercise and medicine like thiazolidiones could improve insulin resistance ${ }^{17-19)}$. Thus early detection of insulin resistance is considered to be important to prevent both diabetes and cardiovascular diseases. In the present study, we showed indexes of insulin resistance were associated with BMI and body fat content. Although advancing age results in the increase of fat mass and the decrease of lean mass under physiological condition 4), insulin resistance indexes were not related to age in this study. These results suggest that, at least in non-diabetes Japanese male population, the development of obesity and fat deposition are associated with the increase of insulin resistance regardless of aging.

Next, we explored the association of insulin resistance indexes with the parameters for the diagnosis of metabolic syndrome. Insulin resistance has been reported to present even in the highnormal blood pressure categories of nonobese and nondiabetic Japanese individuals ${ }^{20)}$. In the present study, we showed that both systolic and diastolic blood pressures were significantly associated with insulin resistance indexes, though the average of blood pressure was not hypertensive. Furthermore, QUICKI and HOMAIR were strongly related with both PG and IRI concentrations at any time point during $75 \mathrm{~g}$ OGTT, though the subjects were not defined as diabetes mellitus. These results suggest the simultaneous development of hypertension and diabetes mellitus in accordance with the increase of insulin resistance.

Dyslipidemia is another risk factor for cardiovascular disease, and the criterion for metabolic syndrome includes high TG and low HDL-C levels. However, the criterion for the diagnosis of metabolic syndrome does not include high TC or LDL-C. We here found that both QUICKI and HOMA-IR were associated with TG and HDL-C levels, but not with TC or LDL-C. In addition, nonHDL-C was not related to QUICKI or HOMA-IR, either. These results suggest us that insulin resistance is associated mainly with serum TG and HDL-C levels even when they are not diagnosed as dyslipidemia, and the increase of plasma LDL-C concentration might be independent risk factor from insulin resistance.

QUICKI and HOMA-IR are both defined by fasting PG and IRI, and are considered to reflect insulin resistance ${ }^{9,10)}$. In Japanese population, there are several reports saying the good correlation of QUICKI and insulin resistance in type 2 diabetes patients ${ }^{21,22)}$. In addition, QUICKI is also useful indicator of the development of insulin resistance even in nonobese, nondiabetic subjects with high-normal blood pressure and in metabolically obese, normal-weight subjects with normal glucose tolerance ${ }^{20,23)}$. We here showed that QUICKI was better associated with the parameters of metabolic syndrome, which is known to be closely associated with the development of insulin resistance ${ }^{15)}$. The criterion for metabolic syndrome revealed to have an additive predictive impact on carotid atherosclerosis as well as Framingham risk score ${ }^{24)}$, and the intervention with diet, exercise and medication could reduce insulin resistance, which results in the reduction of cardiovascular event ${ }^{2,25)}$. These observations suggest us that early evaluation of insulin resistance by QUICKI contributes to the prevention of cardiovascular event due to the impaired glucose tolerance and diabetes mellitus.
Our study has several limitations. First, we could not measure the waist circumference or the area of intra-abdominal fat estimated by computed tomography of enrolled subjects. So, we cannot assess the association between the amount of intraabdominal adipose tissue and insulin resistance. Second, the number of subjects is small and the range of the age is rather narrow in order to fully assess the aging-dependent increase of insulin resistance. Third, both HOMA-IR and QUICKI depend on fasting PG and IRI concentrations, and reflect the relationship between insulin resistance and insulin secretion, suggesting that not only insulin resistance but also $\beta$-cell function might affect both indexes. In addition, QUICKI is recognized as simply being $\log$ HOMA-IR and cannot give quite new information than HOMA-IR ${ }^{26)}$. Forth, the information about the association between insulin resistance indexes and other predictors for cardiovascular event, such as high sensitive C-reactive protein or adiponectin is lacking. Further examination would be required.

In conclusion, these results suggest that insulin resistance was significantly related with the progress of metabolic syndrome even in non-diabetes Japanese male population. Early evaluation of insulin resistance by QUICKI would be expected to contribute to the prevention of cardiovascular event due to metabolic syndrome. 


\section{References}

1) Defornzo RA. Lilly lecture 1987. The triumvirate: beta-cell, muscle, liver. A collusion responsible for NIDDM. Diabetes 37: 667-687, 1988.

2) Reaven GM. Insulin resistance, the insulin resistance syndrome, and cardiovascular disease. Panminerva Med 47: 201-210, 2005.

3) Morimitsu LK, Fusaro AS, Sanchez VH, et al. Fibrinolytic dysfunction after gestation is associated to components of insulin resistance and early type 2 diabetes in latino women with previous gestational diabetes. Diabetes Res Clin Pract 78: 340-348, 2007.

4) Barbieri M, Rizzo MR, Manzella D et al. Age-related insulin resistance: is it an obligatory finding? The lesson from healthy centenarians. Diabetes Metab Res Rev 17: 19-26, 2001.

5) Defronzo RA, Tobin JD, Andres R. Glucose clamp technique: a method for quantifying insulin secretion and resistance. Am J Physiol 237: E214-E223, 1979.

6) Reaven GM, Lithell H, Landsberg L. Hypertension and associated metabolic abnormalities-the role of insulin resistance and the sympathoadrenal system. N Engl J Med 334: 374-381, 1996.

7) Scherrer U, Sartori C. Insulin as a vascular and sympathoexciatory hormone: implications for blood pressure regulation, insulin sensitivity, and cardiovascular morbidity. Circulation 96: 4104-4113, 1997.

8) Serrano Rios M. Relationship between obesity and the increased risk of major complications in non-insulin-dependent diabetes mellitus. Eur J Clin Invest 28(Suppl 2): 14-18, 1998.

9) Matthews DR, Hosker JP, Rudenski AS, et al. Homeostasis model assessment: insulin resistance and beta-cell function from fasting plasma glucose and insulin concentrations in man. Diabetologia 28: 412-419, 1985.

10) Katz A, Nambi SS, Mather K et al. Quantitative insulin sensitivity check index. A simple, accurate method for assessing insulin sensitivity in humans. J Clin Endocrinol Metab 85: 2402-2410, 2000.

11) Isomaa $B$, Almgren $P$, Tuomi $T$ et al. Cardiovascular morbidity and mortality associated with the metabolic syndrome. Diabetes Care 24: 683-689, 2001.

12) Alberti KG, Zimmet P, Shaw J. Metabolic syndrome--a new worldwide definition. A Consensus Statement from the International Diabetes Federation. Diabet Med 23: 469-480, 2006.

13) Lorenzo C, Williams K, Hunt KJ, et al. The National Cholesterol Education Program - Adult Treatment Panel III, International Diabetes Federation, and World Health Organization definitions of the metabolic syndrome as predictors of incident cardiovascular disease and diabetes. Diabetes Care 30: 8-13, 2007.

14) WHO Expert Consultation. Appropriate body-mass index for Asian populations and its implications for policy and intervention strategies. Lancet 363: 157-163, 2004
15) Lann D, LeRoith D. Insulin resistance as the underlying cause for the metabolic syndrome. Med Clin North Am 91: 1063-1077, 2007.

16) Chen H, Sullivan G, Yue LQ, et al. QUICKI is a useful index of insulin sensitivity in subjects with hypertension. Am J Physiol Endocrinol Metab 284: E804-E812, 2003.

17) Olefsky JM, Saltiel AR. PPAR $\gamma$ and the treatment of insulin resistance. Trends Endocrinol Metab 11: 362-368, 2000.

18) Katsuki A, Sumida Y, Gabazza EC et al. QUICKI is useful for following improvements in insulin sensitivity after therapy in patients with type 2 diabetes mellitus. J Clin Endocrinol Metab 87: 29062908, 2002.

19) Watkins LL, Sherwood A, Felinglos M et al. Effects of exercise and weight loss on cardiac risk factors associated with syndrome X. Arch Intern Med 163: 1889-1895, 2003.

20) Katsuki A, Urakawa H, Gabazza EC et al. Quantitative insulin sensitivity check index is a useful indicator of insulin resistance in Japanese metabolically obese, normal-weight subjects with normal glucose tolerance. Endocr J 52: 253-257, 2005.

21) Yokoyama H, Emoto M, Fujiwara $\mathrm{S}$ et al. Quantitative insulin sensitivity check index and the reciprocal index of homeostasis model assessment in normal range weight and moderately obese type 2 diabetic patients. Diabetes Care 26: 2426-2432, 2003.

22) Yokoyama H, Emoto M, Fujiwara $S$ et al. Quantitative Insulin Sensitivity Check Index and the Reciprocal Index of Homeostasis Model Assessment Are Useful Indexes of Insulin Resistance in Type 2 Diabetic Patients with Wide Range of Fasting Plasma Glucose. J Clin Endocrinol Metab 89: 1481-1484, 2004.

23) Kanauchi M, Yamano S, Kanauchi K, et al. Homeostasis Model Assessment of Insulin Resistance, Quantitative Insulin Sensitivity Check Index, and Oral Glucose Insulin Sensitivity Index in Nonobese, Nondiabetic Subjects with High-Normal Blood Pressure. J Clin Endocrinol Metab 88: 3444-3446, 2003.

24) Teramura M, Emoto M, Araki T et al. Clinical impact of metabolic syndrome by modified NCEP-ATPIII criteria on carotid atherosclerosis in Japanese adults. J Atheroscler Thromb 14: 172178,2007

25) Dormandy JA, Charbonnel B, Eckland DJ et al. Secondary prevention of macrovascular events in patients with type 2 diabetes in the PROactive Study (PROspective pioglitAzone Clinical Trial In macroVascular Events): a randomised controlled trial. Lancet 366: 1279-1289, 2005.

26) Wallace TM, Levy JC, Matthews DR. Use and abuse of HOMA modeling. Diabetes Care 27: 1487-1495, 2004. 